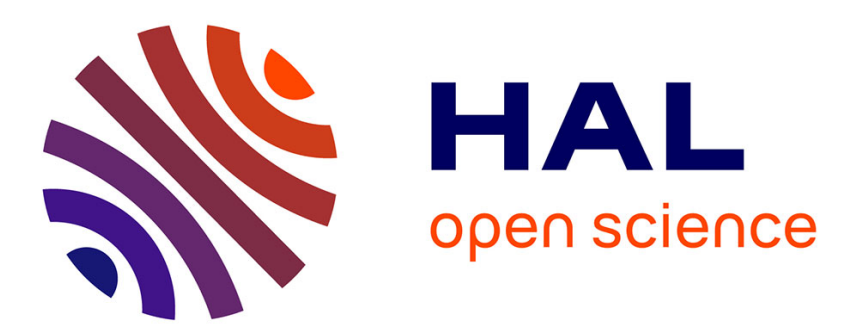

\title{
Doppler Shifted Alpha Transmitter Signals in the Conjugate Hemisphere: DEMETER Spacecraft Observations and Raytracing Modeling
}

\author{
František Němec, O. Santolík, Michel Parrot
}

\section{- To cite this version:}

František Němec, O. Santolík, Michel Parrot. Doppler Shifted Alpha Transmitter Signals in the Conjugate Hemisphere: DEMETER Spacecraft Observations and Raytracing Modeling. Journal of Geophysical Research Space Physics, 2021, 126 (4), pp.e2020JA029017. 10.1029/2020JA029017. insu-03192457

\section{HAL Id: insu-03192457 https://hal-insu.archives-ouvertes.fr/insu-03192457}

Submitted on 8 Apr 2021

HAL is a multi-disciplinary open access archive for the deposit and dissemination of scientific research documents, whether they are published or not. The documents may come from teaching and research institutions in France or abroad, or from public or private research centers.
L'archive ouverte pluridisciplinaire HAL, est destinée au dépôt et à la diffusion de documents scientifiques de niveau recherche, publiés ou non, émanant des établissements d'enseignement et de recherche français ou étrangers, des laboratoires publics ou privés. 


\title{
Doppler Shifted Alpha Transmitter Signals in the Conjugate Hemisphere: DEMETER Spacecraft Observations and Raytracing Modeling
}

\author{
F. Němec ${ }^{1}$, O. Santolík ${ }^{2,1}$, and M. Parrot ${ }^{3}$ \\ ${ }^{1}$ Faculty of Mathematics and Physics, Charles University, Prague, Czech Republic \\ ${ }^{2}$ Department of Space Physics, Institute of Atmospheric Physics of the Czech Academy of Sciences, \\ Prague, Czech Republic \\ ${ }^{3}$ LPC2E/CNRS Orléans, Orléans, France
}

\section{Key Points:}

- DEMETER measurements conjugate to Alpha transmitters show three distinct ways of signal propagation.

- Two of them are identified as ducted and unducted propagation, the third is likely only partially ducted.

- A raytracing analysis is employed to explain the observed Doppler shifts.

Corresponding author: František Němec, frantisek.nemec@gmail.com

This article has been accepted for publication and ${ }^{-1}$ undergone full peer review but has not been through the copyediting, typesetting, pagination and proofreading process, which may lead to differences between this version and the Version of Record. Please cite this article as doi: 10.1029/2020JA029017.

This article is protected by copyright. All rights reserved. 


\section{Abstract}

Alpha navigation transmitters are very low frequency transmitters operating at mid-latitudes, which use a specific discrete radiation pattern at three distinct frequencies $(11.9,12.6$, and $14.9 \mathrm{kHz}$ ). The transmitters are located in the northern hemisphere, but the radiated signals propagate through the magnetosphere to the conjugate hemisphere, where they are detectable by low-altitude spacecraft. We present an analysis of such signals detected by the Detection of Electro-Magnetic Emissions Transmitted from Earthquake Regions (DEMETER) spacecraft at an altitude of about $660 \mathrm{~km}$. It is found that, due to a Doppler shift, the observed signal frequencies can be at times rather different than the radiated frequencies. This indicates wave propagation at large wave normal angles (close to the resonance cone). Simultaneous observations of the same signal with different Doppler shifts reveal three distinct ways of signal propagation: i) ducted propagation, ii) unducted propagation, and iii) propagation interpreted as only partially ducted. A raytracing analysis is employed to obtain typical wave trajectories corresponding to the individual ways of signal propagation and respective Doppler shifts. A reasonable agreement between the observed and calculated Doppler shifts is obtained. Our results demonstrate the peculiarities of very low frequency signal propagation throughout the magnetosphere and the possibility of using Doppler shifts to estimate wave normal angles.

\section{Introduction}

Powerful ground-based very low frequency (VLF) transmitters operating at frequencies between about 10 and $30 \mathrm{kHz}$ are used by military for long distance communications with submerged submarines and for navigation. They can propagate considerable distances in the Earth-ionosphere waveguide (Budden, 1961). Part of the signal power may eventually escape the waveguide and propagate to space, being somewhat attenuated when penetrating through the ionosphere, in particular during the daytime (Cohen et al., 2012; Graf et al., 2013). Having made it to the space environment, the transmitter signals are detectable by low-altitude spacecraft both in the source hemisphere and in the magnetically conjugated region (Cohen \& Inan, 2012; X. Zhang et al., 2017; Zhao et al., 2019). The signals are observable also at larger radial distances in the proximity of given magnetic field lines (Foster et al., 2016; Ma et al., 2017; Koronczay et al., 2018). 
VLF transmitter signals may result in ionospheric heating in the vicinity of transmitters (Bell et al., 2011; Němec et al., 2020), as well as in formation of specific wave events due to wave-wave coupling and triggering (Němec et al., 2017; Gu et al., 2020). They can also interact with energetic electrons in Van Allen radiation belts and eventually result in their precipitation (e.g., Karpman \& Shklyar, 1977; Abel \& Thorne, 1998; Selesnick et al., 2013), as was demonstrated by the analysis of precipitating electron fluxes measured at low altitudes (Gamble et al., 2008; Sauvaud et al., 2008). The efficiency of the transmitter induced pitch angle scattering is generally different for ducted and unducted propagation (Rodger et al., 2010; Ross et al., 2019). Nevertheless, it remains unclear how much energy actually propagates ducted or unducted. At least for low L-shell transmitters, a significant portion of the wave energy may propagate unducted (Clilverd et al., 2008; Z. Zhang et al., 2018). However, at higher L-shells, the waves are possibly primarily ducted, as suggested by the lack of significant wave power above the electron half gyrofrequency for inter-hemispherically propagating waves (Clilverd et al., 2008).

Ducted propagation should correspond primarily to low wave normal angles, while large wave normals are expected for unducted propagation in the magnetically conjugated hemisphere (Bortnik et al., 2006). The two propagation types could be thus in principle distinguished by evaluating the wave normal angles. However, this requires multicomponent magnetic field measurements, which are typically not available with a sufficient frequency resolution at frequencies well above $10 \mathrm{kHz}$. When the wave normal angles are high and close to the resonance cone, the refractive index is large and the signal is detected onboard a low altitude spacecraft with a noticeable Doppler shift (Starks et al., 2009). This allows to distinguish between the ducted and unducted propagation paths, and eventually to estimate the wave normal angle variation with the spacecraft location.

We use electromagnetic wave data obtained by a low-altitude Detection of ElectroMagnetic Emissions Transmitted from Earthquake Regions (DEMETER) spacecraft to analyze Doppler shifts of Alpha navigation transmitter signals detected in the conjugate hemisphere. We distinguish between the ducted and unducted propagation, and we speculate about the respective propagation paths. The used data set is presented in section 2 and the observational results are presented in section 3 . Wave trajectories calculated using a raytracing routine and their consistency with the observed Doppler shifts are eval- 
uated in section 4 . The obtained results and the overall picture are discussed in section 5 . Section 6 contains a brief summary of the main findings.

\section{Data Set}

DEMETER was a French low-altitude spacecraft which operated between 2004 and 2010. It had a circular orbit with an altitude of about $660 \mathrm{~km}$, which was nearly Sunsynchronous. The measurements were thus performed at local times either close to 10:30 hours ("daytime", north-to-south orbits) or 22:30 hours ("nighttime", south-to-north orbits). Survey mode electromagnetic wave measurements were active nearly continuously at geomagnetic latitudes lower than about 65 degrees. In the VLF range (up to $20 \mathrm{kHz}$ ), these consisted of onboard calculated power spectra of one electric (Berthelier et al., 2006) and one magnetic (Parrot et al., 2006) field component. The frequency resolution of the data was about $20 \mathrm{~Hz}$, and the time resolution was about $2 \mathrm{~s}$. Additionally, higher resolution data were available during sporadically active Burst mode intervals. These consisted of waveforms of one electric and one magnetic field component sampled at $40 \mathrm{kHz}$. However, some signals analyzed in the present study are mainly electrostatic and their magnetic field intensity is very low. They are thus generally undetectable in the magnetic field measurements, and only the electric field data are used.

The Alpha navigation system consists of three transmitters: Krasnodar $\left(45.4^{\circ} \mathrm{N}\right.$, $\left.38.2^{\circ} \mathrm{E}\right)$, Novosibirsk $\left(55.8^{\circ} \mathrm{N}, 84.4^{\circ} \mathrm{E}\right)$ and Khabarovsk $\left(50.1^{\circ} \mathrm{N}, 136.6^{\circ} \mathrm{E}\right)$ which transmit specific discrete periodic patterns. Three different frequencies are used by each of the transmitters, equal to about $11,905 \mathrm{~Hz}, 12,649 \mathrm{~Hz}$, and 14,881 Hz. A 0.4 s long pulse at a given frequency is always followed by a $0.8 \mathrm{~s}$ long gap, and then a pulse of a different frequency is radiated. The entire cycle thus takes $3.6 \mathrm{~s}$, and then the sequence is repeated again (Cohen \& Inan, 2012).

\section{Observations}

\subsection{Daytime (North-to-South) Orbits}

An example of a Survey mode frequency-time spectrogram of power spectral density of electric field fluctuations measured during a single DEMETER half-orbit at longitudes close to an Alpha navigation transmitter is shown in Figure 1. The data were obtained on 1 March 2010 during a daytime half-orbit, when the spacecraft moved from 
Figure 1. Frequency-time spectrogram of power spectral density of electric field fluctuations measured on 1 March 2010 during a daytime half-orbit, when the spacecraft moved from north to south. The signals from Alpha navigation transmitters can be identified at frequencies of about $11.9,12.6$, and $14.9 \mathrm{kHz}$, both in the northern and southern hemispheres. Additionally, the signals in the southern hemisphere apparently split to form a V-like shape. The white bars at the top indicate the time intervals when the Burst mode was active and high resolution data were available. The white arrow indicates the magnetic latitude of the Khabarovsk transmitter.

north to south. Alpha navigation transmitter signals at frequencies of about 11.9, 12.6, and $14.9 \mathrm{kHz}$ are identifiable. During the first half of the plotted time interval, the spacecraft was in the northern hemisphere, i.e., in the hemisphere of the transmitters. The signals are limited to the individual transmitted frequencies and they peak at the time when the spacecraft passes close to the transmitter. The white arrow in Figure 1 indicates the magnetic latitude of the Khabarovsk transmitter which is likely the source of the observed signal, as we show below.

The situation in the southern hemisphere, i.e., in the hemisphere geomagnetically conjugated, is more complicated. The signals at individual transmitted frequencies are still observable, albeit with a somewhat lower intensity. Additionally, an apparent gradual decrease of the signal frequencies followed by their subsequent relaxation back to the transmitted values, forming a V-like shape, is observed toward the end of the plotted time interval. It is noteworthy that the $\mathrm{V}$-shapes at higher frequencies are observed slightly sooner (at lower geomagnetic latitudes) than the V-shapes at lower frequencies. The highest frequency V-shape is observed about $13 \mathrm{~s}$ sooner than the middle frequency V-shape, which is followed by the lowest frequency V-shape after about $6 \mathrm{~s}$. The corresponding differences in geomagnetic latitude are lower than one degree. A more detailed view of the situation obtained using the Burst mode data is shown below in Figure 3. Note also that the intense vertical lines observable in the plot correspond to lightning generated sferics/whistlers, and they are not related to the topic of the present paper. There were two Burst mode intervals during the analyzed half-orbit, whose exact times are marked by the white bars at the top of the plot.

Figure 2a shows a geographic map of the situation during the time interval plotted in Figure 1. The projection of the satellite path on the ground is shown by the red 
Figure 2. (a) Geographic map showing the satellite orbit for the time interval from Figure 1 (red curve) and the locations where the Burst mode was active (red bars). The direction of the orbit is marked by the blue arrow. The black crosses in the northern hemisphere correspond to the Alpha transmitter locations, while the black crosses in the southern hemisphere correspond to their geomagnetic conjugates. (b) Average daytime power spectral density of electric field fluctuations at the frequency of $11,905 \mathrm{~Hz}$ is color coded as a function of the geographic location according to the color scale on the right-hand side. (c) The same as (b), but for the frequency of $12,649 \mathrm{~Hz}$. (d) The same as (b), but for the frequency of $14,881 \mathrm{~Hz}$.

curve. The red bars mark the locations where the Burst mode was active. The direction of the orbit (north to south) is marked by the blue arrow. The black crosses in the northern hemisphere show the locations of Alpha navigation transmitters (Novosibirsk and Khabarovsk). The black crosses in the southern hemisphere show their geomagnetic conjugates. Given the satellite path, it seems reasonable to assume that the observed signals come from the Khabarovsk transmitter. The geomagnetic longitudinal separation between the satellite and the Khabarovsk transmitter is lower than about 10 degrees during the entire half-orbit.

Figures $2 \mathrm{~b}-\mathrm{d}$ show geographical maps of average daytime power spectral densities of electric field fluctuations at respective transmitter frequencies. The entire DEMETER data set was used when constructing the plot. The central frequencies considered are marked in each of the figures. The frequency bandwidth of about $60 \mathrm{~Hz}$ was used (3 frequency bins of the instrument). The crosses mark again the locations of the Alpha navigation transmitters and their geomagnetic conjugates. The average wave intensities at all the three analyzed frequencies are significantly increased both above the transmitter locations and close to their geomagnetic conjugates. It is apparent that the waves detected during the example orbit from Figures 1 and 2a indeed come from the Khabarovsk transmitter.

A more detailed view of the two time intervals from Figure 1 obtained using the available Burst mode data is shown in Figure 3. Figure 3a shows a frequency-time spectrogram of power spectral density of electric field fluctuations corresponding to the first Burst mode time interval. During this interval the spacecraft was in the northern hemisphere and rather close to the transmitter. The discrete transmission pattern consist- 
Figure 3. (a) Frequency-time spectrogram of power spectral density of electric field fluctuations corresponding to the first Burst mode time interval from Figure 1, when the spacecraft was located in the northern hemisphere. The discrete transmission pattern of the Alpha navigation transmitters can be identified at the three frequencies (about 11.9, 12.6, and $14.9 \mathrm{kHz}$ ). (b) The same as (a), but for the second Burst mode time interval, when the spacecraft was located in the southern hemisphere. Transmitter signals with gradually decreasing and later increasing frequencies are observed in addition to the expected constant-frequency signals.

ing of $0.4 \mathrm{~s}$ long pulses alternating in frequency can be seen. The observed signal frequencies correspond well to the frequencies radiated by the transmitter. The occasional vertical lines over the plotted frequency range are lightning generated sferics/whistlers, not of importance for the present analysis.

Figure 3b shows a frequency-time spectrogram of power spectral density of electric field fluctuations corresponding to the second Burst mode time interval. This time the spacecraft was in the southern hemisphere close to the region geomagnetically conjugate to the transmitter location. Discrete transmission pattern at the three frequencies can be again identified. Additionally, there are bands of comparatively lower frequencies associated with each of the radiated frequencies. These appear first to decrease in frequency. After reaching a frequency minimum, they gradually increase in frequency again, forming V-like shapes. The frequency minima at higher frequencies occur at earlier times (lower geomagnetic latitudes). Just before reaching the frequency minima, each radiated transmitter frequency $f_{0}$ is observed to be apparently split into three distinct frequencies $f_{o b s}$.

The signals at frequencies lower than the transmitted signal frequency can be interpreted in terms of a Doppler shift of the original transmitted signal frequency:

$$
f_{o b s}=f_{0}\left(1-\frac{\vec{n} \cdot \vec{v}}{c}\right)
$$

where $\vec{n}$ is a refractive index vector (directed along the wave vector), $\vec{v}$ is the DEMETER velocity vector, and $c$ is the speed of light. Considering that $v \approx 7.5 \mathrm{~km} / \mathrm{s}$ and the maximum observed Doppler shifts are as large as about $2 \%$, the corresponding re- 
fractive indices have to be very large, close to 1000 . This is possible for large wave normal angles, close to the resonance cone.

The apparent splitting of each radiated frequency into three distinct frequency bands can be then interpreted as the radiated signal propagating to the spacecraft over three different propagation paths. Each of these eventually results in a different wave normal angle at the observation point, a different refractive index vector (both in direction and magnitude), and thus also a different Doppler shift. The propagation path resulting in principally zero Doppler shift can be interpreted as ducted propagation of the signal, with wave vectors oriented roughly along the ambient magnetic field. The values of refractive index corresponding to such a propagation are quite small and the angle between the wave vector and the satellite velocity vector is rather large. The expected Doppler shift is thus very small (Starks et al., 2009).

Identifying the two remaining propagation paths is less straightforward. There is a propagation path with the Doppler shift increasing toward larger geomagnetic latitudes. This path ceases to exist at very large geomagnetic latitudes and it can be attributed to an unducted propagation. The unducted signals reach the conjugate hemisphere with quite oblique wave normals and exhibit a frequency-dependent latitudinal threshold beyond which they cannot reach (Starks et al., 2009). The threshold latitude decreases with an increasing wave frequency (Shklyar et al., 2010). This can explain why at higher frequencies the bottoms of the V-like shapes are observed closer to the magnetic equator. The identification of the remaining propagation path is the most challenging. It allows the signals to propagate to large latitudes in the opposite hemisphere at not-too-large wave normals (small Doppler shifts). We suggest that it corresponds to only partially ducted propagation. This is supplemented by a raytracing analysis in section 4 .

\subsection{Nighttime (South-to-North) Orbits}

Before progressing to the raytracing analysis, it is instructive to provide an additional experimental supporting argument for the Doppler shift explanation of the observed signals. During nighttime half-orbits the spacecraft moved in an opposite direction as compared to daytime half-orbits. Assuming that the propagation of the signals remains at least qualitatively similar, one might thus expect the Doppler shifts observed during the night to be opposite than during the day. This is indeed the case, as demonstrated 
Figure 4. Frequency-time spectrogram of power spectral density of electric field fluctuations measured on 28 February 2010 during a nighttime half-orbit, when the spacecraft moved from south to north. Although background intensities are quite high due to lightning generated whistlers, the signals from Alpha navigation transmitters can be identified at frequencies of about $11.9,12.6$, and $14.9 \mathrm{kHz}$, both in the northern and southern hemispheres. Additionally, the signal at the frequency of about $14.9 \mathrm{kHz}$ observed in the southern hemisphere apparently splits to form an inverted V-like shape. The white bars at the top indicate the time intervals when the Burst mode was active and high resolution data were available.

Figure 5. (a) Geographic map showing the satellite orbit for the time interval from Figure 4 (red curve) and the locations where the Burst mode was active (red bars). The direction of the orbit is marked by the blue arrow. The black crosses in the northern hemisphere correspond to the Alpha transmitter locations, while the black crosses in the southern hemisphere correspond to their geomagnetic conjugates. (b) Average power spectral density of electric field fluctuations at the frequency of $11,905 \mathrm{~Hz}$ is color coded as a function of the geographic location according to the color scale on the right-hand side. (c) The same as (b), but for the frequency of 12,649 Hz. (d) The same as (b), but for the frequency of 14,881 Hz.

by an example event in Figure 4. The format of the figure is the same as the format of Figure 1. The data were obtained on 28 February 2010 during a nighttime half-orbit, when the spacecraft moved from the south to north. Although the spectrogram is quite noisy due to lightning generated whistlers, increased wave intensities at the three distinct frequencies corresponding to the Alpha transmitters are identifiable. During the second half of the plotted time interval, the observed signal frequencies closely match the transmitted frequencies. This time the spacecraft was in the northern hemisphere, i.e., in the hemisphere of the transmitters. In the first half of the plotted time interval, the observed frequencies closely match the transmitted frequencies only for the two lower frequency signals (11.9 and $12.6 \mathrm{kHz})$. A considerable increase of the signal frequency is apparent for the highest frequency $(14.9 \mathrm{kHz})$ signal. The absence of the Doppler shifted, i.e., (partially) unducted, signals at lower frequencies can be explained by a magnetospheric reflection. The nighttime lower-hybrid frequency above the spacecraft is higher than the signal frequencies, resulting in their reflection above the satellite altitude (Shklyar et al., 2004; Vavilov et al., 2013). 
Figure 6. (a) Frequency-time spectrogram of power spectral density of electric field fluctuations corresponding to the first Burst mode time interval from Figure 4, when the spacecraft was located in the southern hemisphere. The discrete transmission pattern of the Alpha navigation transmitters can be identified at the three frequencies (about 11.9, 12.6, and $14.9 \mathrm{kHz}$ ). Additionally, a gradually increasing signal frequency is observed in the upper left part of the figure. (b) The same as (a), but for the second Burst mode time interval, when the spacecraft was located in the northern hemisphere.

Figure 5 uses the same format as Figure 2 to depict the situation during the example nighttime half-orbit and nighttime intensity maps. During nighttime half-orbits the spacecraft moved in a northward direction, as marked by the blue arrow in Figure 5a. The longitudes of the example orbit are between the longitudes of the Novosibirsk and Khabarovsk transmitters marked by the black crosses. The transmitter locations and the average intensity maps in Figures 5b-d indicate that in the beginning of the example time interval (i.e., in the southern hemisphere) the spacecraft likely detects predominantly the signal from the Khabarovsk transmitter. On the other hand, in the end of the example time interval (i.e., in the northern hemisphere) the spacecraft likely detects predominantly the signal from the Novosibirsk transmitter. Nevertheless, all the Alpha transmitters radiate at the same frequencies and comparable latitudes, only with a different time coding. The exact transmitter detected at a given time is thus not important for this study. Note that another bright dot of statistically increased signal levels occurs above the NWC transmitter located at North West Cape, Australia $\left(21.8^{\circ} \mathrm{S}, 114.2^{\circ} \mathrm{E}\right)$ and operating at $19.8 \mathrm{kHz}$, owing to spectral broadening (Xia et al., 2020).

The example half-orbit contains two Burst mode intervals for which high-resolution frequency-time spectrograms can be obtained. These are depicted in Figure 6. Figure 6a corresponds to earlier times when the spacecraft was located in the southern hemisphere (i.e., conjugate to the transmitter). It shows the time coded signals at frequencies of about 11.9 and $12.6 \mathrm{kHz}$, which directly correspond to the transmitted signal. The signal at a frequency of about $14.9 \mathrm{kHz}$ is partly obscured by strong natural (lightning related) background emissions. However, the signal apparently splits and extends to higher frequencies around the middle of the plotted time interval, forming an inverted V-like shape. This is particularly clear in the left part of the plot, where the background emissions are 
weaker and the transmitter signal is more pronounced. An indication of this transmitter signal shift to higher frequencies is identifiable also after 14:06:30 UT, corresponding to the second half of the inverted V-like shape. Note that the original signal at the frequency of $14.9 \mathrm{kHz}$ does not disappear during the time of the inverted V-like shape. It still remains identifiable, albeit being arguably weaker. Note also that no such deviation of the signal frequencies from the transmitted frequencies is observable for signals at 11.9 and $12.6 \mathrm{kHz}$, in agreement with the Survey mode plot from Figure 4. Figure 6b, shown for completeness, depicts the situation in the transmitter hemisphere. The signals at frequencies of about $11.9,12.6$, and $14.9 \mathrm{kHz}$ are identifiable despite the strong natural background related to the lightning activity. They correspond directly to the frequencies transmitted by the Alpha navigation transmitters.

\section{Raytracing Analysis}

Having suggested the Doppler shift as an explanation of the frequency-time properties of the observed transmitter signals, we devote this section to a more quantitative analysis. Ducted signals, which propagate along the ambient magnetic field with not too large wave normals, have too low refractive indices and do not result in observable Doppler shifts. They can be thus identified as the signals in the conjugate hemisphere at frequencies corresponding to the transmitted frequencies. As such, their interpretation is straightforward and they are not further discussed in this section. Instead, we focus on the interpretation of the signals at frequencies apparently departing from the transmitted frequencies, which we interpret as unducted or only partially ducted signals.

We use a raytracing analysis which allows us to follow individual ray trajectories from the northern hemisphere to the conjugated hemisphere and to evaluate the respective wave normal angles and refractive indices upon reaching the DEMETER altitudes. The used raytracing code is the same as the one used formerly by, e.g, Santolík et al. (2016) and Martinez-Calderon et al. (2020). A simple dipole geomagnetic field model is used. The density is modeled assuming a diffusive equilibrium with a reference altitude of $670 \mathrm{~km}$, ion and electron temperatures $1000 \mathrm{~K}$, and plasma number density at the reference altitude $18,000 \mathrm{~cm}^{-3}$ with $92 \%$ of oxygen ions and $8 \%$ of hydrogen ions. The propagation takes place well within the plasmasphere, so that no plasmapause density drop is considered in the density model. Note, however, that the exact choice of the density model does not significantly affect the raytracing results. The important point is that the max- 
Figure 7. Example raytracing trajectories corresponding to an unducted signal propagation. The signals were started at geomagnetic latitudes $33.4^{\circ}, 41.4^{\circ}$, and $49.4^{\circ}$, roughly corresponding to the geomagnetic latitude of the Khabarovsk transmitter and geomagnetic latitudes of $\pm 8^{\circ}$ from it. The waves were started directly upwards (perpendicular to the ground surface) at an altitude of $110 \mathrm{~km}$, as indicated by the short black arrows. The blue, green, and red curves were obtained for wave frequencies of $11,905 \mathrm{~Hz}, 12,649 \mathrm{~Hz}$, and 14,881 Hz, respectively. The black dashed curves show the respective dipolar magnetic field lines corresponding to individual starting points.

imum lower hybrid frequencies along the propagation paths are lower than all the considered signal frequencies $(11,905 \mathrm{~Hz}, 12649 \mathrm{~Hz}$, and 14,881 Hz), corresponding to a typical daytime situation. This is not the case during the night, when the maximum lower hybrid frequencies along the propagation paths are typically higher than the lower two signal frequencies. They thus get magnetospherically reflected and do not reach the DEMETER altitudes in the conjugate hemisphere (Chum et al., 2009; Shklyar et al., 2010; Vavilov et al., 2013). This explains why during the nighttime half-orbits the (partially) unducted signals are observed only at the highest frequency .

Let us start the discussion with purely unducted signals, whose Doppler shifts observed by DEMETER were formerly analyzed by Starks et al. (2009). We assume that, before exiting to higher altitudes, the transmitter generated signals propagate some distance within the Earth-ionosphere waveguide. Referring to the maps of mean wave intensities in Figures 2b-d, the signals are considered to exit the waveguide within 8 degrees of the geomagnetic latitude of the transmitter (about $41.4^{\circ}$ ). Note that we limit the calculation to the magnetic meridian of the transmitter, but principally the same picture holds also for nearby geomagnetic longitudes. Considering that the refractive index within the Earth-ionosphere waveguide is close to 1, a wave exiting to the ionosphere bends according to the Snell's law toward the normal. It is thus reasonable to start the raytracing assuming vertical wave normals at altitudes of about $110 \mathrm{~km}$ (Helliwell, 1965).

The calculated ray trajectories for three different starting geomagnetic latitudes $\left(33.4^{\circ}, 41.4^{\circ}\right.$, and $\left.49.4^{\circ}\right)$ are shown in Figure 7 . The black dashed curves show the dipolar magnetic field lines corresponding to individual starting points. The blue, green, and red color curves show the trajectories obtained for the wave frequencies of 11,905 Hz, 12,649 Hz, 
Figure 8. (a) Wave normal angles corresponding to unducted trajectories from Figure 7 at the times when the emissions reach DEMETER altitudes in the southern hemisphere are plotted as a function of the respective final geomagnetic latitudes. The blue, green, and red curves were obtained for wave frequencies of $11,905 \mathrm{~Hz}, 12,649 \mathrm{~Hz}$, and $14,881 \mathrm{~Hz}$, respectively. The horizontal dashed lines show the wave normal angles of respective resonance cones. (b) The same as (a), but for refractive index values.

and $14,881 \mathrm{~Hz}$, respectively. It can be seen that the waves starting at larger geomagnetic latitudes end at larger geomagnetic latitudes (i.e., the calculated ray trajectories do not intersect anywhere). However, there is a clear upper geomagnetic latitude limit for the ending points of the propagation paths (Starks et al., 2009; Shklyar et al., 2010). Additionally, higher frequency waves end at lower geomagnetic latitudes than lower frequency waves started at the same geomagnetic latitude.

In order to understand the observed Doppler shifts, it is crucial to consider the refractive index value and wave vector direction upon reaching the DEMETER altitudes in the conjugate hemisphere. It is found that the wave vectors are typically rather close to the resonance cone, being oriented toward larger L-shells, i.e., approximately southward. This corresponds well to the observed Doppler shifts. During the daytime, when the spacecraft moves from north to south, the satellite velocity is oriented roughly along the wave vectors. The Doppler shift then results in observed frequencies being lower than the transmitted frequencies. During the nighttime, the spacecraft moves from south to north. The satellite velocity is thus oriented roughly opposite to the wave vectors, and the Doppler shift results in observed frequencies being larger than the transmitted frequencies. As the wave vectors are close to the resonance cone, the values of the refractive index are high and the Doppler shift significant.

Wave normal angles and refractive index values as a function of the geomagnetic latitude in the conjugate hemisphere are analyzed more in detail in Figures $8 \mathrm{a}$ and 8b, respectively. These were obtained by tracing the waves started within a full range of considered geomagnetic latitudes in the northern hemisphere until they reach the DEMETER altitudes in the southern hemisphere. The blue, green, and red color curves again correspond to individual transmitter signal frequencies. The horizontal dashed lines in Figure $8 \mathrm{a}$ show the respective resonance cone angles. Given that the waves propagate 
Figure 9. Example raytracing trajectories corresponding to only a partially ducted signal propagation. The waves were started at a geomagnetic latitude of $49.4^{\circ}$, i.e., approximately $8^{\circ}$ northward from the Khabarovsk transmitter. They were assumed to propagate ducted, i.e., exactly along the geomagnetic field line all the way to the geomagnetic equator. They are then traced from the equator to the southern hemisphere, with wave normal angles of $\pm 70^{\circ}$, assuming the wave vectors lie in the meridional plane. The blue, green, and red curves were obtained for wave frequencies of $11,905 \mathrm{~Hz}, 12,649 \mathrm{~Hz}$, and 14,881 Hz, respectively. The black dashed curves show the dipolar magnetic field lines.

from north to south, the wave normal angles are larger than 90 degrees. However, they gradually decrease and approach the resonance cone as the final geomagnetic latitudes approach a given threshold value. Correspondingly, the refractive index (and thus also the Doppler shift) gradually increases and eventually diverges at the threshold final geomagnetic latitude. This threshold latitude is different for each wave frequency, being slightly closer to the geomagnetic equator for higher frequency waves. The unducted propagation can thus explain the left-hand parts of V-like shapes in Figure 1 and the righthand part of the inverted V-like shape in Figure 4.

The remaining problem is how to explain the right-hand parts of V-like shapes in Figure 1 and the left-hand part of the inverted V-like shape in Figure 4. These are formed by the signals which make it to larger geomagnetic latitudes than the unducted propagation threshold. Moreover, their refractive index decreases with increasing geomagnetic latitude. As both the purely ducted and purely unducted signals have been already discussed and identified in the frequency-time spectrograms, we need to look for a possible third way of the signal propagation. We suggest that the signal might be ducted only for a part of the propagation path. Significant Doppler shifts are observed upon reaching the conjugate hemisphere, i.e., the final wave normal angles are close to the resonance cone. The signal is thus clearly unducted when reaching the spacecraft. One could consider many different ducted/unducted propagation combination schemes. However, in order to keep things reasonably simple, we assume the signal to be ducted all the way to the geomagnetic equator, and unducted farther. The upper frequency limit on the wave ducting in the ducts of an enhanced density is $0.5 f_{c e} \cos \theta_{k}$ (Smith, 1961), where $\theta_{k}$ is the wave normal angle and $f_{c e}$ the electron cyclotron frequency. Moreover, even the waves 
Figure 10. The same as Figure 8, but for the propagation scheme from Figure 9.

with frequencies below the frequency limit or those ducted in density troughs are less likely to be ducted when the limit is lower. It is thus reasonable to assume that at least more oblique waves can become unducted close to the magnetic field minimum at the geomagnetic equator. We further assume a rather large range of wave normal angles leaving the duct (Woodroffe et al., 2013), ranging from $\theta_{k}=-70^{\circ}$ to $\theta_{k}=70^{\circ}$. The unducted propagation of such waves to the conjugate hemisphere is calculated using the raytracing routine. The results obtained for the field line about $8^{\circ}$ northward from the Khabarovsk transmitter are depicted in Figure 9. The wave trajectories for the waves with the two extreme initial wave normal angles (i.e., $\theta_{k}= \pm 70^{\circ}$ ) are shown, and the color coding again represents the three different signal frequencies.

It can be seen that the waves reach the DEMETER altitudes in the conjugate hemisphere close to the respective magnetic field line. However, unlike the situation of the purely unducted propagation, their final wave normal angles are close to the resonance cone at lower geomagnetic latitudes, and they become more field aligned at larger geomagnetic latitudes. This is demonstrated in Figure 10, which depicts the results obtained by tracing the waves started with a full range of considered wave normal angles $\left(-70^{\circ} \leq\right.$ $\left.\theta_{k} \leq 70^{\circ}\right)$. The used format is the same as in Figure 8. The results correspond to the Doppler shifts being the largest at not-so-large geomagnetic latitudes and progressively decreasing toward larger geomagnetic latitudes. This is exactly the behavior observed for the third type of the signal propagation. We note that waves leaving the duct with large wave normal angles are crucial to get very close to the resonance cone at low geomagnetic latitudes. Should the waves leaving the duct be limited to lower wave normal angles $\left(-35^{\circ} \leq \theta_{k} \leq 35^{\circ}\right)$, only the parts of the dependences plotted by thick curves in Figure 10 would be obtained. We also note that a group of waves launched with wave vectors directed toward the Earth with proper wave normal angles reaches DEMETER altitudes with wave normal angles far from the resonance cone. These are not shown in Figure 10, as they extend beyond the plotted wave normal angle range. However, such waves do not result in considerable Doppler shifts and are not of importance for the present analysis. 
The time delays corresponding to the interhemispheric ducted propagation are on the order of a few tenths of second. When the wave normal angle upon arrival is not too close to the resonance cone, the respective time delays are very close to those obtained for the ducted propagation. This is the case for the unducted propagation at lower geomagnetic latitudes and for the partially ducted propagation at larger geomagnetic latitudes. However, the time delays increase considerably when the signals propagate close to the resonance cone, and they diverge when the final wave normal angle approaches the resonance. This explains the considerable time delays of individual transmitter pulses observed for the largest Doppler shifts in Figures 3b and 6a.

\section{Discussion}

Electromagnetic wave measurements performed by the low-altitude DEMETER spacecraft allow us to observe signals from powerful ground-based VLF transmitters. Unfortunately, only a single electric and a single magnetic field components are measured, which does not allow us to perform a detailed wave analysis and to directly experimentally determine the wave normal angles. Moreover, as the magnetic field component of the analyzed signals is typically too weak to be detected by the used instrumentation, the observations are limited to a single electric field component.

Doppler shifts due to the spacecraft movement are typically rather small and difficult to identify, unless the refractive index becomes comparatively large. This is, however, the case of whistler waves propagating with wave normal angles close to the resonance cone. Then, the Doppler shifts are readily observable and their magnitude can be used to estimate the refractive index.

The observations performed in the hemisphere conjugate to the Alpha navigation transmitters revealed that during specific times/at some spacecraft locations, a given transmitter signal may split to up to three signals at different frequencies. These can be interpreted in terms of different propagation paths of the signal, resulting in different wave normal angles, refractive indices, and Doppler shifts at the observation point. The signal without an observable Doppler shift can be attributed to a ducted propagation, which occurs at low wave normal angles well away from the resonance cone. The signal with the Doppler shift steadily increasing with geomagnetic latitude until reaching a given latitudinal (frequency-dependent) threshold can be interpreted as an unducted signal (Starks 
et al., 2009). The used raytracing analysis confirms this interpretation, achieving at least a qualitative agreement with observations.

A rather surprising finding is the third propagation path necessary to explain the third observed signal frequency. We suggested that the corresponding signal might be ducted in the beginning and become eventually unducted close to the equatorial region. Under such a propagation scheme, the raytracing results and the predicted Doppler shifts are in agreement with the observations. Most importantly, the Doppler shift steadily decreasing with increasing geomagnetic latitude is obtained. The wave has to be assumed to be initially ducted along a magnetic field line slightly northward from the transmitter location (i.e., at a larger L-shell). There may be two possible reasons why this should be the case. First, the ionospheric attenuation is lower at larger geomagnetic latitudes (Helliwell, 1965), so that one might expect more VLF transmitter power to penetrate through in there. Second, and probably more important, the wave ducting depends crucially on the electron cyclotron frequency. It becomes less likely for low electron cyclotron frequencies (Smith, 1961), suggesting that the waves tend to get unducted at larger Lshells close to the geomagnetic equator. This propagation scheme requires the waves to leave the duct with large range of wave normal angles. This may arguably correspond to the real situation. However, the large range of initial wave normal angles considered at a given location can also account for the waves leaving the duct over a range of latitudes/Lshells, which must be the case in reality.

Achieving an exact quantitative match between the calculated and observed Doppler shifts is difficult, as the wave propagation at large wave normal angles is very sensitive to even small variations of the conditions. However, the obtained qualitative agreement suggests that our interpretation of the three propagation paths is indeed correct. It is further supported by the fact that as the spacecraft moves in different directions during the daytime and nighttime half-orbits, the signs of the Doppler shifts are expected to be different, corresponding to the observations. Finally, it is worth to note again that in order to reach the DEMETER altitudes in the conjugate hemisphere the signal frequencies have to be higher than the maximum lower hybrid frequencies along the respective propagation paths. This immediately explains why the Doppler shifted signals are not observed at lower frequencies during the night, when the lower hybrid frequencies are higher (Chum et al., 2009; Shklyar et al., 2010; Vavilov et al., 2013). 


\section{Conclusions}

We presented an analysis of electromagnetic wave signals from VLF Alpha navigation transmitters detected in the conjugate hemisphere by the low-altitude DEMETER spacecraft. The observed signal frequencies sometimes significantly differ from the transmitted frequencies, which can be explained by a Doppler shift. Considering the magnitudes of the observed Doppler shifts, the Doppler shifted signals need to propagate with high refractive indices (i.e., close to the resonance cone). A given signal frequency is sometimes observed to split into three distinct frequencies, indicating three different ways of signal propagation between the hemispheres. The first of them, resulting in principally zero Doppler shifts, corresponds to a ducted propagation at low wave normal angles. It is the only propagation path which allows signals at frequencies below the maximum lower hybrid frequency to reach low altitudes in the conjugate hemisphere. The second way of propagation was identified as unducted propagation, characterized by a maximum (frequency dependent) latitudinal threshold reachable by the propagating signal. As demonstrated by the raytracing analysis and consistent with the observed Doppler shifts, the final wave normal angles steadily increase with increasing geomagnetic latitude, reaching the resonance cone at the latitudinal threshold. The third way of propagation was interpreted as only a partially ducted propagation, with the waves becoming unducted close to the geomagnetic equator. The corresponding calculated wave normal angles decrease with geomagnetic latitude. This propagation path allows a propagation to larger latitudes than the purely ducted situation, in agreement with the observations. Our results show the possibility of using Doppler shifts to estimate wave normal angles/propagation paths of VLF signals throughout the inner magnetosphere and demonstrate the peculiarities of the signal propagation therein.

\section{Acknowledgments}

We thank the engineers from CNES and scientific laboratories (CBK, IRAP, LPC2E, LPP, and SSD of ESTEC) who largely contributed to the success of the DEMETER mission. DEMETER data are accessible from the https://sipad-cdpp.cnes.fr website. F. N. and O. S. acknowledge the support of GACR Grant 21-01813S.

\section{References}

Abel, B., \& Thorne, R. M. (1998). Electron scattering loss in the Earth's inner mag- 
netosphere: 1. dominant physical processes. J. Geophys. Res., 103(A2), 23852396. doi: 10.1029/97JA02919

Bell, T. F., Graf, K., Inan, U. S., Piddyachiy, D., \& Parrot, M. (2011). DEMETER observations of ionospheric heating by powerful VLF transmitters. Geophys. Res. Lett., 38(L11103). doi: 10.1029/2011GL047503

Berthelier, J. J., Godefroy, M., Leblanc, F., Malingre, M., Menvielle, M., Lagoutte, D., ... Pfaff, R. (2006). ICE, the electric field experiment on DEMETER. Planet. Space Sci., 54, 456-471.

Bortnik, J., Inan, U. S., \& Bell, T. F. (2006). Temporal signatures of radiation belt electron precipitation induced by lightning-generated MR whistler waves: 1 . methodology. J. Geophys. Res., 111(A02204). doi: 10.1029/2005JA011182

Budden, K. G. (1961). The wave-guide mode theory of wave propagation. London, Great Britain: Logos Press.

Chum, J., Santolik, O., \& Parrot, M. (2009). Analysis of subprotonospheric whistlers observed by DEMETER: A case study. J. Geophys. Res., 114(A02307). doi: 10 $.1029 / 2008 \mathrm{JA} 013585$

Clilverd, M. A., Rodger, C. J., Gamble, R., Meredith, N. P., Parrot, M., Berthelier, J.-J., \& Thomson, N. R. (2008). Ground-based transmitter signals observed from space: Ducted or nonducted? J. Geophys. Res., 113(A04211). doi: 10.1029/2007JA012602

Cohen, M. B., \& Inan, U. S. (2012). Terrestrial VLF transmitter injection into the magnetosphere. J. Geophys. Res., 117(A08310). doi: 10.1029/2012JA017992

Cohen, M. B., Lehtinen, N. G., \& Inan, U. S. ～(2012). Models of ionospheric VLF absorption of powerful ground based transmitters. Geophys. Res. Lett., 39(L24101). doi: 10.1029/2012GL054437

Foster, J. C., Ericksson, P. J., Baker, D. N., Jaynes, A. N., Mishin, E. V., Fennel, J. F., .. Kanekal, S. G. (2016). Observations of the impenetrable barrier, the plasmapause, and the VLF bubble during the 17 March 2015 storm. $\quad J$. Geophys. Res. Space Physics, 121, 5537-5548. doi: 10.1002/2016JA022509

Gamble, R. J., Rodger, C. J., Clilverd, M. A., Sauvaud, J.-A., Thomson, N. R., Stewart, S. L., , . Berthelier, J.-J. (2008). Radiation belt electron precipitation by man-made VLF transmissions. J. Geophys. Res., 113(A10211). doi: 10.1029/2008JA013369 
Graf, K. L., Lehtinen, N. G., Spasojevic, M., Cohen, M. B., Marshall, R. A., \& Inan, U. S. (2013). Analysis of experimentally validated trans-ionospheric attenuation estimates of VLF signals. J. Geophys. Res. Space Physics, 118, 2708-2720. doi: 10.1002/jgra.50228

Gu, W., Chen, L., Xia, Z., An, X., \& Horne, R. B. (2020). Alpha transmitter signal reflection and triggered emissions. Geophys. Res. Lett., 47(e2020GL090165). doi: $10.1029 / 2020$ GL090165

Helliwell, R. A. (1965). Whistlers and related ionospheric phenomena. Stanford Univ. Press, CA.

Karpman, V. I., \& Shklyar, D. R. (1977). Particle precipitation caused by a single whistler-mode wave injected into the magnetosphere. Planet. Space Sci., 25, 395-403. doi: 10.1016/0032-0633(77)90055-1

Koronczay, D., Lichtenberger, J., Juhász, L., Steinbach, P., \& Hospodarsky, G. (2018). VLF transmitters as tools for monitoring the plasmasphere. J. Geophys. Res. Space Physics, 123, 9312-9324. doi: 10.1029/2018JA025802

Ma, Q., Mourenas, D., Li, W., Artemyev, A., \& Thorne, R. M. (2017). VLF waves from ground-based transmitters observed by the Van Allen Probes: Statistical model and effects on plasmaspheric electrons. Geophys. Res. Lett., 44, 6483-6491. doi: 10.1002/2017GL073885

Martinez-Calderon, C., Němec, F., Katoh, Y., nad C. Kletzing, K. S., Hospodarsky, G., Santolik, O., .. Kurkin, V. I. (2020). Spatial extent of quasiperiodic emissions simultaneously observed by Arase and Van Allen Probes on 29 november 2018. J. Geophys. Res. Space Physics, 125, e2020JA028126. doi: 10.1029/2020JA028126

Němec, F., Pekař, J., \& Parrot, M. (2020). NWC transmitter effects on the nightside upper ionosphere observed by a low-altitude spacecraft. J. Geophys. Res. Space Physics, 125(e2020JA028660). doi: 10.1029/2020JA028660

Němec, F., Č́žžek, K., Parrot, M., Santolík, O., \& Záhlava, J. (2017). Line radiation events induced by very low frequency transmitters observed by the DEMETER spacecraft. J. Geophys. Res. Space Physics, 122, 7226-7239. doi: 10.1002/2017JA024007

Parrot, M., Benoist, D., Berthelier, J. J., Blecki, J., Chapuis, Y., Colin, F., ... Zamora, P. (2006). The magnetic field experiment IMSC and its data pro- 
cessing onboard DEMETER: Scientific objectives, description and first results. Planet. Space Sci., 54, 441-455.

Rodger, C. J., Carson, B. R., Cummer, S. A., Gamble, R. J., Clilverd, M. A., Green, J. C., .. Parrot, M. (2010). Contrasting the efficiency of radiation belt losses caused by ducted and nonducted whistler-mode waves from ground-based transmitters. J. Geophys. Res., 105(A12208). doi: 10.1029/2010JA015880

Ross, J. P. J., Meredith, N. P., Glauert, S. A., Horne, R. B., \& Clilverd, M. A. (2019). Effects of VLF transmitter waves on the inner belt and slot region. $J$. Geophys. Res. Space Physics, 124, 5260-5277. doi: 10.1029/2019JA026716

Santolík, O., Parrot, M., \& Němec, F. (2016). Propagation of equatorial noise to low altitudes: Decoupling from the magnetosonic mode. Geophys. Res. Lett., 43, 6694-6704. doi: 10.1002/2016GL069582

Sauvaud, J.-A., Maggiolo, R., Jacquey, C., Parrot, M., Berthelier, J.-J., Gamble, R. J., \& Rodger, C. J. (2008). Radiation belt electron precipitation due to VLF transmitters: Satellite observations. Geophys. Res. Lett., 35(L09101). doi: 10.1029/2008GL033194

Selesnick, R. S., Albert, J. M., \& Starks, M. J. (2013). Influence of a ground-based VLF radio transmitter on the inner electron radiation belt. J. Geophys. Res. Space Physics, 118, 628-635. doi: 10.1002/jgra.50095

Shklyar, D. R., Chum, J., \& Jiřiček, F. (2004). Characteristic properties of Nu whistlers as inferred from observations and numerical modelling. Ann. Geophys., 22, 3589-3606. doi: 10.5194/angeo-22-3589-2004

Shklyar, D. R., Parrot, M., Chum, J., Santolík, O., \& Titova, E. E. $\quad$ (2010). On the origin of lower- an upper-frequency cutoffs on wedge-like spectrograms observed by DEMETER in the midlatitude ionosphere. J. Geophys. Res., 115(A05203). doi: 10.1029/2009JA014672

Smith, R. L. (1961). Propagation characteristics of whistlers trapped in field-aligned columns of enhanced ionization. J. Geophys. Res., 66(11), 3699-3707. doi: 10 $.1029 / J Z 066 \mathrm{i} 011 \mathrm{p} 03699$

Starks, M. J., Bell, T. F., Quinn, R. A., Inan, U. S., Piddyachiy, D., \& Parrot, M. (2009). Modeling of Doppler-shifted terrestrial VLF transmitter signals observed by DEMETER. Geophys. Res. Lett., 36(L12103). doi: 10.1029/2009GL038511 
Vavilov, D. I., Shklyar, D. R., Titova, E. E., \& Parrot, M. ～（2013). ～Study of the lower hybrid resonance frequency over the regions of gathering earthquakes using DEMETER data. J. Atm. Solar-Terr. Phys., 100-101. doi: 10.1016/j.jastp.2013.03.019

Woodroffe, J. R., Streltsov, A. V., Vartanyan, A., \& Milikh, G. M. (2013). Whistler propagation in ionospheric density ducts: Simulations and DEMETER observations. J. Geophys. Res. Space Physics, 1-8. doi: 10.1002/2013JA019445

Xia, Z., Chen, L., Zhima, Z., \& Parrot, M. ～(2020). $\quad$ Spectral broadening of NWC transmitter signals in the ionosphere. Geophys. Res. Lett., $47(\mathrm{e} 2020 \mathrm{GL} 088103)$. doi: 10.1029/2020GL088103

Zhang, X., Zhao, S. F., Ruzhin, Y., Liu, J., \& Song, R. (2017). The spatial distribution of three Alpha transmitter signals at the topside ionosphere. Radio Sci., 52, 653-662. doi: 10.1002/2016RS006219

Zhang, Z., Chen, L., Li, X., Xia, Z., Heelis, R. A., \& Horne, R. B. (2018). Observed propagation route of VLF transmitter signals in the magnetosphere. J. Geophys. Res. Space Physics, 123, 5228-5537. doi: 10.1029/2018JA025637

Zhao, S., Zhou, C., Shen, X., \& Zhima, Z. (2019). Investigation of VLF transmitter signals in the ionosphere by ZH-1 observations and full-wave simulation. $J$. Geophys. Res. Space Physics, 124, 4697-4709. doi: 10.1029/2019JA026593 